\title{
Politica internacional, nacional y local: la gestión pública de la accesibilidad espacial para las personas con discapacidad
}

\section{International, national and local policy: public management of spatial accessibility for people with disabilities}

\author{
Johana Linares Garcia (iD
}

Universidad Industrial de Santander

johana.linares.garcia@gmail.com

\author{
Andrea Hernández-Quirama \\ Universidad Industrial de Santander \\ ahernanq@uis.edu.co
}

\section{Héctor Mauricio Rojas Betancur iD}

Universidad Industrial de Santander

hmrojasb@uis.edu.co

\begin{abstract}
Resumen
En el presente artículo se desarrolla una reflexión sobre la interrelación entre apropiación territorial, accesibilidad, espacio y movilidad como elementos que determinan la inclusión social de los grupos poblacionales que integran una ciudad y comparten un territorio. El estudio se concentra en las personas con discapacidad, las cuales buscan su desarrollo autónomo y reclaman sus posibilidades de participación. A partir de los diferentes instrumentos públicos para el ordenamiento territorial, se realiza un análisis para la ciudad de Bucaramanga (Colombia), centrado en el cumplimiento de los marcos normativos que promueven una política pública de inclusión de las personas con discapacidad en el pleno desarrollo de sus posibilidades de participación social.
\end{abstract}

Palabras clave: Discapacidad, inclusión social, territorio, ciudad.

\begin{abstract}
A reflection on the interrelationship between territorial appropriation, accessibility, mobility and space develops as elements that determine the social inclusion of the population groups that make up a city and share a territory, in particular persons with disabilities, who are looking for their autonomous development and claim their chances of participation. Based on the different public instruments for land-use planning, an analysis is carried out for the city of Bucaramanga, Colombia, focused on the implementation of regulatory frameworks that promote a public policy of inclusion of the people with disabilities in the development of their possibilities for social participation.
\end{abstract}

Keywords: Social inclusion, disability, territory, city.

Articulo: Recibido el 2 de marzo de 2019 y aprobado el 23 de octubre de 2019.

Cómo citar este articulo:

Linares-García, J., Hernández-Quirama, A. \& Rojas-Betancur, H. (2019). Politica internacional, nacional y local: la gestión pública de la accesibilidad especial para las personas con discapacidad. Reflexión Politica 21 (43), pp. 142154. doi: $10.29375 / 01240781.3522$ 


\section{Introducción}

Discapacidad es un concepto que se ha ido enriqueciendo a lo largo del tiempo gracias a los ejercicios de comprensión individuales y colectivos, especialmente desde la singularidad de los individuos que integran las ciudades. Existen cuatro discursos básicos de comprensión: el médico rehabilitador, el socialemancipatorio, el integrador y el de la diversidad funcional.

Desde el modelo del médico-rehabilitador se concebían a las personas con alguna discapacidad física o mental como personas enfermas y, por ende, no aptas para participar en la vida social. En ese momento, se atribuía la responsabilidad al individuo que no tenía las capacidades para integrarse porque se consideraba que se hallaba incompleto, siendo las incapacidades situaciones imperfectas, en consecuencia, sujetas a una desviación respecto de cierta normalidad. De este modo, atender la discapacidad era un asunto que requería un tratamiento médico individual, enfocado a superar el déficit fisiológico (Rodríguez y Ferreira 2010, pp. 290, 292). No obstante, la discapacidad también es una interacción entre la sociedad y los sujetos que desborda el ámbito institucional normativo de la salud (Brogna, 2006).

Durante los años 60 surge el discurso social-emancipatorio impulsado por los colectivos hacia la promoción de una vida independiente que reivindica el derecho a la autonomía, en donde se afirma que la discapacidad no es un asunto individual sino social, dado que es la sociedad la que, al no comprender la diversidad de sus individuos, los margina y excluye de la participación en la vida cotidiana (Rodríguez y Ferreira 2010, p. 292-293). Al respecto, Dear. et al., (1997) plantea que a lo diferente se le imponen límites espaciales que permiten la separación, desde las diferencias percibidas y construidas entre lo mismo o lo "normal" y "lo otro" (p.457).

En esta medida, el contexto social "contribuye a la construcción de un determinado sentido, y unas ciertas prácticas asociadas, de la discapacidad; un contexto en el que los espacios físicos son inadecuados y los estereotipos discriminatorios" (Rodríguez y Ferreira 2010, p.293). De tal forma, se construye a partir de los 80 un discurso crítico en que la discapacidad se inserta en el modelo socialemancipatorio, enfocándose en la integración social. Este discurso integrador plantea que la inclusión se puede lograr siempre y cuando se vuelque la mirada a las alteraciones en la interacción entre las personas y el medio; alteraciones que son ocasionadas por ese entorno social (CEPAL, 2014, p.5).

La discapacidad se ha entendido, entonces, como un término que "incluye déficits, limitaciones en la actividad y restricciones en la participación. Indica los aspectos negativos de la interacción entre un individuo (con una "condición de salud") y sus factores contextuales (factores ambientales y personales)" (OMS, 2001, p.206).

No obstante, en Europa, algunos movimientos sociales han buscado redefinirla como diversidad funcional, aunque este discurso no erradica las profundas desigualdades sociales, la marginación y la exclusión. Asimismo abandona la naturaleza material e imperfecta del cuerpo, puesto que las personas con discapacidad serán siempre la evidencia de que tenemos que rechazar esa pretensión de poder existir con independencia de nuestro cuerpo (Rodríguez y Ferreira, 2010). Un reflejo de esto, se basa en la necesidad de acudir a recursos técnicos y tecnológicos que buscan la normalización. Desdibujar la diversidad implica negar la materialidad del ser, aquella que ha sido una fuente enriquecedora del conocimiento y desarrollo. Estos modelos han impactado a las políticas públicas y de desarrollo territorial que han pasado de la rehabilitación a la inclusión. En Colombia, hablar de diversidad funcional es un reto dado que el medio físico aún no brinda las condiciones necesarias para que los sujetos logren apropiarse del territorio.

Lograr la apropiación territorial es un asunto complejo, en la medida en que el espacio debe ser accesible y permitir "la circulación, utilización, orientación, seguridad y funcionalidad" (Olivera, 2006, p.332). Existe entonces, una estrecha relación entre apropiación territorial, espacio, accesibilidad y movimiento (o movilidad). 
El espacio, ha sido definido desde diversas disciplinas como la geografía, la sociología, el urbanismo, la ingeniería y psicología (Olivera, 2006, p.333). Para Lefebvre (citado por Martínez, 2013), el espacio es una construcción social en la medida en que este resulta de las interacciones sociales, las prácticas y experiencia cotidianas. El espacio debe considerarse, por tanto, un producto que se consume, que se utiliza, pero que no es como los demás objetos producidos, ya que él mismo interviene en la producción (p.14).

Para el caso de las personas con discapacidad, las relaciones sociales son determinantes en el acceso y la permanencia en los espacios educativos (Moriña y Melero, 2016, p.51). "De esta manera, el espacio social es vital en la medida en que permite este encuentro relacional al envolver las cosas que se producen y comprender las relaciones en su coexistencia y simultaneidad: en su orden y/o desorden" (Lefebvre, 1969, p.129). Por lo tanto, se dota de sentido a partir del "reconocimiento de los vínculos entre individuo y sociedad; [...] en él, confluyen relaciones de carácter funcional, de interdependencia, de selección, de reproducción, de sustitución o de cambio, cuya actuación se refleja en diferentes escalas, niveles y tiempos" (Santos, 2000 [citado por Celia, 2001, p.380]).

Uno de los más notables autores que han contribuido a la comprensión del espacio es el geógrafo brasileño Milton Santos, quien en su obra La naturaleza del espacio, expresa cómo esta conceptualización varía dependiendo el momento histórico. Para él, las observaciones y resultados del espacio "parten de un sistema descriptivo y cualitativo de las relaciones sociales y su entorno" (Santos, 2000 citado por Celia, 2001, p. 379).

Aunado a lo anterior, es importante comprender que la ciudad per se, es un espacio creado (Lefebvre, 1969, p.130). Hablar del espacio en la ciudad, es hablar del espacio como un bien público cuya accesibilidad debe garantizarse a todos los miembros de la sociedad, así como fijar sus condiciones de uso y actividades. Para Habermas, la ciudad es sobre todo el espacio público en donde el poder se hace visible, en donde la sociedad se fotografia, en donde el simbolismo colectivo se materializa. La ciudad es un escenario que cuanto más abierto esté a todos, más expresará la democratización política y social (Borja y Muxí, 2003, p.19).
Borja, en La ciudad conquistada (2003) expresa que el espacio puede ser concebido como "un instrumento de redistribución social, de cohesión comunitaria, de autoestima colectiva [...] es espacio político, de formación y expresión de voluntades colectivas, el espacio de la representación, pero también del conflicto” (p.29).

Respecto a los conflictos que pueden generarse en el espacio, estos se hallan vinculados con la accesibilidad y la movilidad. Para Olivera (2006), la accesibilidad espacial está determinada por las barreras y accesos urbanos que impiden garantizar la igualdad de oportunidades. Se define entonces como "la facilidad con que un lugar puede ser alcanzado desde otro, es una cualidad referida al espacio" (Olivera, 2006, p.332). Las barreras en el espacio "generan retraimiento, sedentarismo, no inclusión en los beneficios de vivir en sociedad y, por tanto, una forma de exclusión: las personas se convierten en prisioneras de su espacio, cautivas en su hogar. Planificar un espacio libre de barreras o eliminar las existentes no es solo un ejercicio de solidaridad con las personas con discapacidad [...] permite mayor autonomía e independencia, por tanto, mayor calidad de vida" (Abellán y Olivera, 2004, p.186).

Por su parte, la movilidad se une a la accesibilidad en la medida en que "da cuenta de las posibilidades de acceso a los servicios básicos para el desarrollo de la vida social, para la participación activa en las actividades económicas, y para la sociabilización de las personas" (Blanco, et al., 2014, p.3), es por tanto una práctica social necesaria para el desarrollo autónomo que pone en juego las necesidades de los sujetos de realizar sus actividades de reproducción de la vida social en un cierto contexto territorial y temporal.

Gutiérrez define la movilidad como "una práctica social de viaje que conjuga deseos y necesidades de desplazamiento (que en conjunto pueden definirse como requerimientos de movilidad) y capacidades de satisfacerlos. De su interacción resultan las condiciones de accesibilidad de grupos sociales, sea de sí mismos o de sus bienes" (Blanco, et al., 2014, p.3) La misma autora señala que esta definición enfatiza: 1) que una práctica de viaje es una práctica en el territorio; 2) que una práctica implica una frecuencia de realización de un comportamiento de viaje; 3) que una práctica social de viaje implica la reiteración de comportamientos que definen un patrón (pauta o modelo) de 
desplazamiento en un contexto social, espacial y temporalmente determinado (Blanco et al., 2014, p.3). De esta manera, la accesibilidad, el espacio y la movilidad determinan la apropiación territorial de un individuo.

La apropiación territorial o territorialidad es un concepto que permite comprender la ciudad, dado que esta se constituye en gran medida por las acciones de los sujetos a través de la historia (Echeverría,2001,p.220).Existen, entonces, diversos actores que marcan el territorio: públicos, privados, comunitarios o sujetos de grupos poblacionales vulnerables, como las personas con discapacidad. "Si bien todos éstos marcan simultáneamente el territorio, algunos logran mayores o menores grados de expresión y consolidación y definición de las prácticas territoriales y relaciones espaciales" (Echeverría y Rincón, 2000, pp. 12-13).

Pero ¿qué es la ciudad? Al respecto existen múltiples conceptualizaciones. Para el presente caso se acudirá a las reflexiones de Borja y Muxí (2003) en donde "ciudad" puede ser entendida como "un lugar con mucha gente. Un espacio público, abierto y protegido. Un lugar es decir un hecho material productor de sentido. Una concentración de puntos de encuentros" (p.13). De esta manera, la ciudad requiere, como primera medida, el desarrollo y la consolidación de espacios colectivos. David Harvey (2008), aborda el derecho a la ciudad como aquel que va más allá del acceso a lo que ya existe y comprende la necesidad de participar en la construcción de ciudad, para hacerla y rehacerla acorde a los deseos de las personas que la habitan y comprendiendo su singularidad (p.17). Como afirma De Lorenzo (2007), la ampliación de las posibilidades de participación de toda la población extiende los sentidos de apropiación y marca un gran avance en la comprensión de las limitaciones particulares.

La ciudad brinda, entre otras cuestiones, la posibilidad de acceder a la garantía de derechos. Acorde a los planteamientos de Borja y Muxí (2003), toda intervención en el espacio debe considerar la heterogeneidad social que por su esencia "favorece lo imprevisible, introduce desorden y hace más posible la innovación" (p.20). De esta manera, el ordenamiento territorial, al ser "la expresión espacial de las políticas económicas, sociales, culturales y ecológicas de la sociedad" (Pujadas y Font, 1998, p.11), incluye la transformación del espacio y crea territorialidad en la medida en que permite el aglutinamiento social y la creación de identidades (Borja y Muxí, 2003, p.5). Por ende, el ordenamiento se halla en estrecha relación con el espacio público y el hecho de que este "sea [un] elemento determinante de la forma de la ciudad ya es razón para atribuirle el rol ordenador del urbanismo y en primer lugar de la trama urbana" (Borja, 2003, p.137).

El ordenamiento y la apropiación de espacio público están básicamente condicionados por el uso y la interacción cotidiana de todas las personas que, desde sus propios lugares y sentidos, participan de esta interacción. No solo es un proceso técnico, también debe consultar y comprender las propias construcciones cotidianas de los sujetos que la pueblan, entendiendo que esta producción territorial es muy dinámica en el tiempo y puede tener diferentes escalas, de manera que, en un mismo espacio, pueden existir diferentes capas territoriales que interactúan constantemente (Baquero, 2016, p.12). Así, al analizar el espacio público con el objetivo de proponer recomendaciones al ordenamiento territorial implica analizar "la redistribución del espacio, los usos del suelo, los procesos de ocupación y la infraestructura del espacio" (Mendoza, 2013, p.48).

La presente reflexión se centra en el análisis de estos elementos a partir de la revisión de los marcos normativos a nivel internacional y nacional, así como su cumplimiento a través de instrumentos de gestión pública y territorial a nivel local, identificando retos para la inclusión de personas con discapacidad en la participación social y construcción de ciudad.

\section{Discapacidad: un asunto de Derechos Humanos}

La discapacidad se ha convertido con los años en un tema de especial atención a nivel internacional y nacional. En el presente apartado, se reflexiona acerca de cómo la discapacidad llega a considerarse un asunto de Derechos Humanos y en qué medida esto se ve reflejado a nivel normativo, en términos de accesibilidad, así como en las políticas de desarrollo $y$, por ende, en el ordenamiento territorial de la ciudad de Bucaramanga, ubicada en el noreste de Colombia.

Las Guerras Mundiales provocaron el aumento de las discapacidades adquiridas y, por lo tanto, el crecimiento del interés de rehabilitar a 
aquellos veteranos que habían sufrido afectaciones mediante políticas sociales, cuestión que luego se extendió a las demás personas, sin importar el origen de su condición (González, 2010, p.17). Desde las Naciones Unidas, se atiende el asunto como una cuestión susceptible a prevenir y rehabilitar. Se proponen, entonces, programas especialmente dirigidos a personas con dificultades visuales y de movilidad (ONU, s. f.). No obstante, no es sino hasta la década de los 70 que, gracias a la presión de los grupos sociales con discapacidad, la movilidad empieza a considerarse un asunto de Derechos Humanos, reflejando un cambio en el enfoque para su atención y tratamiento, y buscando la inclusión a partir de la garantía de derechos como el empleo, la educación, la vivienda y la atención sanitaria; para, de esta forma, permitir el desarrollo autónomo de los individuos.

La Declaración de los Derechos de los Impedidos, adoptada por la Asamblea General el 9 de diciembre de 1975, fomenta la protección de estos derechos a nivel nacional e internacional. Se reconocía el hecho de que los discapacitados debían gozar de los mismos derechos políticos y civiles que los demás, incluyendo medidas que les permitiesen ser autosuficientes. La Declaración reafirmaba los derechos de estas personas a la educación, a la atención sanitaria y a servicios de colocación, ampliando su espectro a todos aquellos derechos que deben gozar todas las personas: educación, trabajo, salud e incluso el derecho a la alegría y al reconocimiento social (ONU, s. f.).

Según la Organización Mundial de la Salud (2011, p.10), las razones por las que la discapacidad debe considerarse una cuestión de Derechos Humanos responden a la desigualdad que viven estas personas en términos de acceso a la educación, salud, empleo y participación de la vida cotidiana; y por ende, su dignidad y autonomías se ven afectadas. Durante los 90 las reflexiones en torno a la discapacidad llevaron a los países a sancionar leyes en contra de la discriminación en este periodo de tiempo. Uno de los principales logros de las Naciones Unidas en la década fue la declaración emitida por la Asamblea General de las Normas Uniformes, de la igualdad de oportunidades para las personas con discapacidad (Resolución 48/96). Aunque no se trata de un instrumento jurídicamente vinculante, las Normas Uniformes representan el firme compromiso moral y político de los gobiernos con respecto de la adopción de medidas encaminadas a lograr la igualdad de oportunidades para las personas con discapacidad. Las Normas son un instrumento para la formulación de políticas, y sirven como base para la cooperación técnica y económica (ONU, s. f.).

Allí, entre las esferas previstas para garantizar la igualdad en la participación de las personas con discapacidad se aborda, entre otros aspectos, la necesidad de brindar posibilidades de acceso al medio físico; así como a la información y comunicación (Naciones Unidas, 1994, pp. 1617). No obstante, no es hasta el primer decenio del siglo XXI en que defensores de los derechos de las personas con discapacidad (PCD) presionan a los países miembros de las Naciones Unidas para concretar una convención internacional que exija el cumplimiento de las responsabilidades de los Estados para buscar la inclusión de este grupo. Este esfuerzo se ve materializado en la Convención sobre los Derechos de las Personas con Discapacidad (CDPD) y su protocolo facultativo, que se aprueba en diciembre del año 2006 y es ratificado por los países miembros a inicios del 2007. En este se realiza una apuesta por cambiar el enfoque de atención de la discapacidad y los Estados adquieren obligaciones como sancionar leyes y tomar las demás medidas administrativas correspondientes en los casos en que sea necesario; modificar o derogar leyes, costumbres o prácticas que, directa o indirectamente, generen discriminación; incluir la discapacidad en todas las políticas y los programas pertinentes; abstenerse de cometer todo acto o práctica que no estén en consonancia con la CDPD; tomar todas las medidas adecuadas para eliminar la discriminación de las personas con discapacidad por parte de cualquier individuo, organización o empresa privada (Naciones Unidas, 2006 [citado por OMS, 2011, p.10]).

Los principios que orientan la Convención son ocho, entre los que se destacan la accesibilidad, expresada en el artículo 3 y desarrollada en el artículo 9, indicando la necesidad de incluir la "identificación y eliminación de obstáculos y barreras de acceso" (Naciones Unidas, 2006, p.10) presentes en el ambiente construido físico (edificios, vías públicas, transporte, viviendas, etc.), a partir del desarrollo, promulgación y supervisión de normas mínimas en los bienes de uso público o de carácter privado con afluencia de personas, permitiendo así que "las personas con discapacidad puedan vivir en forma independiente y participar 
plenamente en todos los aspectos de la vida" (2006).

En esta convención se induce los conceptos de ajustes razonables y diseño universal el primero orientado a realizar modificaciones o adaptaciones en el medio para garantizar el goce y la inclusión de las personas con discapacidad. El segundo demanda la creación de entornos que consideren las diferencias de los seres humanos para acceder al medio físico (Naciones Unidas, 2006, p.5). Estos acuerdos internacionales se han ratificado y se hallan expresados en la Constitución Política y la legislación nacional, especialmente en términos de accesibilidad, tal y como se presenta en el siguiente apartado.

\section{Discapacidad y accesibilidad: de la Constitución Política a la legislación nacional}

Paralelo al desarrollo internacional en materia de Discapacidad, en Colombia se han generado una serie de normas que se fundamentan en los artículos 13, 47, 54 y 68 de la Constitución Política Colombiana (1991), en las cuales se plantea promover condiciones de igualdad, protección a las personas vulnerables, rehabilitación de quienes en ese momento se denominaban "disminuidos físicos, sensoriales y psíquicos” (República de Colombia, 1991, Art. 47), así como garantías laborales y educativas. Un ejemplo de esto es la ley 361 de 1997, en la cual se hace un reconocimiento a los derechos fundamentales de esta población a nivel económico, social y cultural, con el fin de lograr su integración (República de Colombia, 1997). De igual forma, se plantean las condiciones mínimas en materia de accesibilidad para PCD a vías, espacio público, mobiliario urbano, construcción o reestructuración de edificaciones públicas o privadas (Art. 43), cuestiones que son contempladas en la ley 388 del mismo año alrededor de ordenamiento territorial.

La ley 361 de 1998 es considerada una herramienta que busca suprimir y evitar las barreras físicas en los territorios. Se define a la accesibilidad como "la condición que permite en cualquier espacio o ambiente interior o exterior, el fácil y seguro desplazamiento de la población en general y el uso en forma confiable y segura de los servicios instalados en estos ambientes" (Art. 44).

A partir de este momento y tras la ley 762 de 2002, por la cual "se aprueba la Convención
Interamericana de eliminación de todas las formas de discriminación de las PCD" (República de Colombia, 2002), se establecen iniciativas legislativas y decretos reglamentarios para garantizar la accesibilidad espacial de este grupo poblacional. Se define, además, en el artículo 1, a la discapacidad como una "deficiencia física, mental o sensorial, ya sea de naturaleza permanente o temporal que limita la capacidad de ejercer una o más actividades esenciales de la vida diaria, que puede ser causada o agravada por el entorno técnico, económico y social” (República de Colombia, 2002).

Con lo anterior, se observa cómo a pesar de los esfuerzos de articulación con la agenda internacional, en Colombia se entiende la discapacidad como un asunto del sujeto en donde el entorno puede contribuir a reducir su participación autónoma en la vida cotidiana, razón por la cual se deben prevenir o eliminar las barreras que limiten su integración. No obstante, con ello se da impulso a los decretos reglamentarios expedidos por el Ministerio de Transporte y los expedidos por el entonces llamado Ministerio de Ambiente, Vivienda y Desarrollo Territorial. El primero de ellos es el Decreto 1660 expedido por el Ministerio de Transporte (2003). De allí, se adoptan las Normas Técnicas Colombianas (NTC) de accesibilidad como la 4139 (ICONTEC, 2009) y la 6047 (ICONTEC, 2013). Se establecen los modos de accesibilidad y la señalización necesaria en los medios de transporte terrestres y aéreos dirigidos especialmente a las PCD. A su vez, para la aplicación de este decreto, se deben considerar las disposiciones del Ministerio de Ambiente, Vivienda y Desarrollo Territorial expedidas en el Decreto 1538 (2005), en el cual se especifican los lineamientos para garantizar el acceso al espacio público así como a las propiedades. Se incluyen consideraciones con respecto a la textura del suelo y rampas de acceso. En síntesis, se busca la integración de medidas necesarias para la eliminación de las barreras internas y externas de las edificaciones. Llama la atención que este decreto surja para reglamentar de manera parcial la ley 361 de 1997, pues se tardaron aproximadamente seis años para establecer las consideraciones generales con respecto al ambiente construido.

Tras el Decreto 1538, se logra la actualización del Manual de Accesibilidad al Medio Físico y al Transporte (Presidencia de La República 
et al., 2000), dando como resultado la Guía de accesibilidad al espacio público y a edificaciones abiertas y de uso público (Min. Ambiente, Vivienda y Desarrollo, 2008). Aunque a nivel internacional los retos en materia de inclusión se basan desde la perspectiva de derechos, en Colombia se considera esta articulación a partir de la ley 1346 de 2009, por la cual "se aprueba la convención sobre los derechos de las personas con discapacidad" (República de Colombia, 2009), en donde la accesibilidad al medio físico es determinante para garantizar el derecho a la educación, a una vivienda digna, al empleo, al desarrollo autónomo y a la participación en la vida cotidiana. Tras ella, se crea la Guía de diseño accesible y universal (COLDEPORTES, 2009).

Las apuestas de esta ley se consolidan en la ley estatutaria 1618 de, en la cual se busca adoptar "medidas de inclusión, acción afirmativa y de ajustes razonables y eliminando toda forma de discriminación por razón de discapacidad" (República de Colombia, 2013, Art. 1), otorgando la responsabilidad a las entidades públicas y privadas con respecto a la accesibilidad, tal y como se establece en el artículo 14.
Corresponde a las entidades públicas y privadas encargadas de la prestación de los servicios públicos, de cualquier naturaleza, tipo y nivel, desarrollar sus funciones, competencias, objetos sociales, y en general, todas las actividades, siguiendo los postulados del diseño universal, de manera que no se excluya o limite el acceso en condiciones de igualdad, en todo o en parte, a ninguna persona en razón de su discapacidad. para ello, dichas entidades deberán diseñar, implementar y financiar todos los ajustes razonables que sean necesarios para cumplir con los fines del artículo. (República de Colombia, 2013, Art. 14)

En síntesis, la legislación nacional en materia de accesibilidad determina un horizonte común a nivel de desarrollo nacional y, por ende, local. A nivel de ordenamiento territorial, la Ley Orgánica de Ordenamiento Territorial, propone como principios la equidad y el equilibrio territorial, en donde se debe procurar el acceso equitativo a las oportunidades de desarrollo. En la figura 1 se presenta de forma cronológica el desarrollo normativo en el ámbito de la accesibilidad.

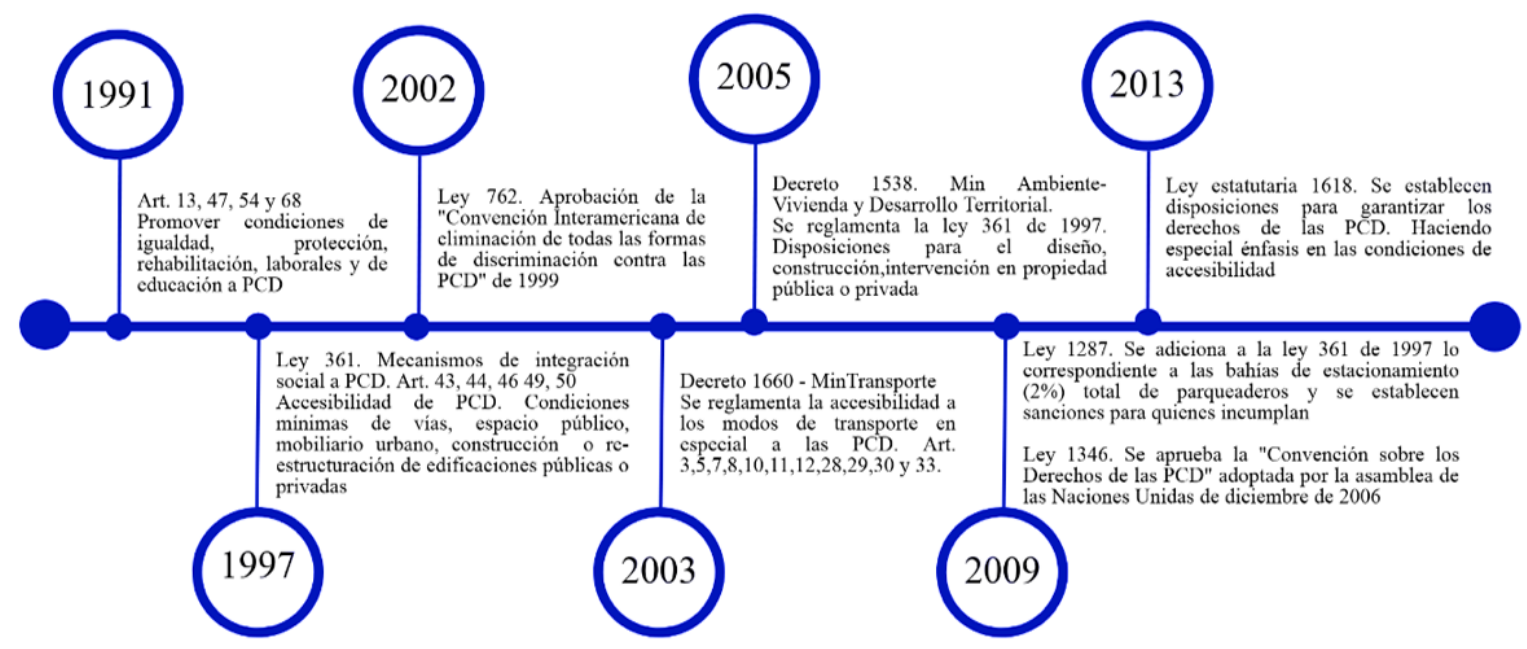

Figura 1. Línea de tiempo normatividad nacional en accesibilidad. Fuente: elaboración propia.

\section{Discapacidad y desarrollo: desde los ODM y los ODS a los Planes de desarrollo nacional, departamental y municipal}

La exclusión social de las personas con discapacidad se halla en estrecha relación con el fenómeno de la pobreza (Cortés y Sotomayor, 2016). De esta manera se vincula la discusión de los derechos de las personas con discapacidad en el marco de los Objetivos de Desarrollo del Milenio (ODM). Según un estudio realizado por el Banco Mundial en el año 2005 y citado por la Naciones Unidas: "Si bien se estima que las personas con discapacidad constituyen un 10\% de la población mundial, se ha sugerido que la discapacidad podría estar asociada con el $20 \%$ de la población mundial que vive en la pobreza" (Naciones Unidas, 2009, p.3). No obstante, "la discapacidad no se menciona explícitamente en 
los Objetivos de Desarrollo del Milenio (ODM) ni en las 21 metas o los 60 indicadores [que contiene]" (OMS, 2011, p.12).

A diferencia de los ODM; los Objetivos de Desarrollo Sostenible (ODS), contemplan metas e indicadores en los objetivos de pobreza, educación de calidad, trabajo decente, reducción de las desigualdades;yciudadesycomunidadessostenibles (Taccari, 2017, pp. 14-15). Particularmente, frente a este último, la ONU en la tercera conferencia Hábitat (2017) centra su atención aportando la "iniciativa de ciudad próspera", en la cual se comprenden la productividad, infraestructura, calidad de vida, equidad e inclusión social, sustentabilidad ambiental, gobernanza y legislación como dimensiones para medir la prosperidad. Es de resaltar que, en la nueva agenda urbana, el tema de la accesibilidad fue transversal (ONU, 2017). Adicionalmente, se promueve la innovación en tecnología al alcance de las PCD y, como estrategia para mejorar los procesos de planeación, diseño urbano y ordenamiento.

Acorde al marco internacional, Colombia ha venido ratificando estos acuerdos y los traduce en la política social a través de los Planes de Desarrollo desplegados en los diferentes niveles territoriales en donde la accesibilidad se considera preponderante al permitir la garantía de los derechos de esta población. El Plan Nacional de Desarrollo (2014-2018) propone, desde el enfoque poblacional fortalecer las políticas públicas. A este respecto, dice:

La Política Pública de Discapacidad e Inclusión Social, que busca cambiar de una visión asistencialista a políticas de desarrollo humano con un enfoque de derechos. Este enfoque permite el acceso a bienes y servicios para las personas con discapacidad, con criterios de pertinencia, calidad y disponibilidad; respeto por la diferencia y su aceptación como parte de la diversidad; accesibilidad; igualdad de oportunidades, y eliminación de prácticas que conlleven a la marginación y segregación de cualquier tipo (Departamento Nacional de Planeación 2015, p.67).

Como se observa, a nivel nacional la discapacidad configura en una Política Social transversal que responde al esfuerzo y a la presión de los grupos sociales a través de los años, y hoy es coordinada por el Ministerio de Salud y Protección Social. Como principios se plantean, entre otros, promover el desarrollo humano, lo cual se puede lograr mediante "el respeto por la diferencia y la accesibilidad universal" (Min. Salud y Protección Social, 2014, p.88). Entre los retos se plantea contar con una perspectiva diferencial, el logro de la equidad, la inclusión social, el acceso y la participación de las personas con discapacidad, partiendo del análisis temporal y espacial de las características, necesidades, potencialidades, tipo de discapacidad, situación en el contexto social y cultural colombiano, dando herramientas prácticas para la protección, atención, cuidado y promoción de tal población e impulsando los ajustes y cambios necesarios para hacer que el contexto sea accesible a nivel educativo económico, laboral, cultural, social, ambiental y arquitectónico. (Min. de Salud y Protección Social, 2014, p.73). Por lo cual, al hablar de accesibilidad, se hace necesario vincularla con la movilidad, el uso y apropiación del espacio (p.101). Para ello, los gobernantes a nivel departamental y municipal deberán promover e implementar ajustes razonables que garanticen la participación de los ciudadanos en la vida cotidiana desde un enfoque de Derechos Humanos.

No obstante, en Santander, las metas establecidas para este tema no contemplan la accesibilidad espacial. En ellas se plantea el acceso a programas y proyectos liderados por la Secretaría de Desarrollo, así como metas dirigidas al fortalecimiento de la respuesta institucional y comunitaria en donde se destacan iniciativas de rehabilitación y fortalecimiento de capacidades. También de promoción de la política pública nacional a nivel departamental y municipal. En cuanto a educación, se plantea la ampliación de becas para el desarrollo de estudios a nivel de primaria y secundaria (Gobernación de Santander, 2017, pp. 227, 250-251).

En Bucaramanga, el Gobierno Municipal crea una serie de programas de atención prioritaria y focalizada a grupos vulnerables, como es el caso de las PCD. Ahora bien, resulta desafortunado encontrar que, aunque el programa dirigido a este grupo tiene como foco la reducción de todo tipo de barreras (entre las que incluyen las físicas y arquitectónicas), sus indicadores de producto no refieren ninguna acción tendiente a ello, y por el contrario se centran en otorgar cupos para la rehabilitación, atención integral, orientación ocupacional, entradas a eventos deportivos, sin considerar la accesibilidad espacial (Alcaldía de Bucaramanga, 2016). 


\section{Discapacidad: directrices de ordenamiento departamental, metropolitano y municipal}

Los sistemas de planificación integral comprenden la articulación entre las apuestas de desarrollo social y económico y las transformaciones territoriales que sean necesarias para alcanzar tal fin. En Santander se cuenta con los Lineamientos y Directrices de Ordenamiento Territorial, en donde a partir del diagnóstico de los sistemas natural, económico, sociocultural, ambiental construido y político-institucional, se establecen ciertas medidas para articular el desarrollo territorial municipal con el departamental. Esto con el ánimo de "[...] garantizar un modelo de desarrollo equitativo, equilibrado, integrado y sostenible, construir municipios con identidad diferenciada, capaces de generar riqueza con el aprovechamiento de sus ventajas particulares, $\mathrm{y}$, como resultado de todo, consolidar un departamento con una vocación clara, capaz de vencer los retos de la globalización y de mejorar la calidad de vida de todos los santandereanos" (Gobernación de Santander, 2014, p.23). Por ende, se hace imperante la necesidad de reducir la pobreza desde un enfoque diferencial, en donde se tienen en cuenta a las personas con discapacidad; buscando aumentar la cobertura en educación superior y garantizando el acceso a estos grupos excluidos, con el ánimo de elevar la calidad de vida de los habitantes (2014, p.286).

Las dinámicas poblacionales en el departamento llevaron, con el tiempo, a concentrar mayor población urbana en el Área Metropolitana de Bucaramanga, conformada por los municipios de Floridablanca, Piedecuesta, Girón y la misma cabecera departamental. Indudablemente, el crecimiento acelerado expone a las ciudades a asumir "fuertes presiones sociales y ambientales" (Gobernación de Santander, 2014, p.171), así como "[conducir] a la macrocefalia urbana y al desequilibrio en el resto del territorio [...] aumenta[r] los problemas de conectividad y por ende, socioeconómicos" (Gobernación de Santander, 2014, p. 71-72).

Aunque en los lineamientos no se habla de un grupo poblacional específico, entre las metas se estima reducir la pobreza del $33 \%$ al $10 \%$, y erradicar la indigencia (que tiene actualmente una tasa del 9\%) (Gobernación de Santander, 2014, p.287), razón por la cual la inclusión de personas con discapacidad es determinante a fin de lograr los objetivos. A nivel metropolitano, desde el año 2011 se planteó la necesidad de mejorar la movilidad y accesibilidad asociadas al transporte sostenible. Se busca desde entonces "garantizar mayor equidad, es decir, generar mejoras prioritariamente para las poblaciones vulnerables" (Área Metropolitana de Bucaramanga, 2011, p.137).

En esta escala de planeación, se promueve la funcionalidad urbana para la competitividad y productividad a través de "[...] un transporte colectivo moderno, seguro y eficiente y la rápida conexión entre los diversos centros de empleo y entre éstos y las zonas de vivienda, con un fácil acceso a los equipamientos sociales, de servicios y económicos. Una excelente oferta y cobertura de una red vial jerarquizada y especializada y de parqueo donde se dé especial importancia a la movilidad peatonal y de otros medios alternativos como parte del proceso de mejoramiento de la calidad de vida" (Área Metropolitana de Bucaramanga, 2011, p.110).

Además del transporte, como estrategia territorial se determina la necesidad de ampliar el espacio público como una iniciativa que permite articular el tejido urbano mediante un sistema verde, teniendo en cuenta al sistema poblacional y su diversidad (Área Metropolitana de Bucaramanga, 2011, p.113). Esto se traduce a nivel municipal en medidas de intervención dirigidas a reducir los problemas de accesibilidad, a mejorar el desplazamiento $\mathrm{y}$, por ende, a aumentar las capacidades de los sujetos para movilizarse; todo ello con el ánimo de garantizar la producción de territorios incluyentes a partir de las experiencias cotidianas de interacciones en el espacio público.

El POT del municipio de Bucaramanga (2014) plantea estrategias alrededor de dos aspectos: transporte y espacio público. Con respecto al transporte, las directrices giran alrededor de la accesibilidad al Sistema Integrado de Transporte Masivo (SITM), a la Metrolínea, y la necesidad de lograr un transporte sostenible buscando el bienestar social a partir de la articulación metropolitana.

El sistema de transporte masivo denominado METROLÍNEA, se conceptualizó como un sistema integral que cubriera los cuatro municipios involucrados, sin que el área de influencia del proyecto fuera afectada por las divisiones territoriales y en el cual los servicios permitieran realizar integraciones físicas $\mathrm{y}$ temporales. $\mathrm{El}$ sistema contempla dentro de su diseño mejorar las condiciones actuales de movilidad de las 
personas (incluyendo las que se encuentran con algún grado de discapacidad) [...] [Como objetivo de transporte sostenible se propone] Garantizar mayor equidad, es decir, generar mejoras en la movilidad y accesibilidad prioritariamente para las poblaciones vulnerables, las cuales se pueden definir con base en aspectos como modo de transporte, ingresos, género edad o discapacidad. (Alcaldía de Bucaramanga, 2014, pp. 43,75).

Aunado a lo anterior, en el POT se establecen las directrices sobre las zonas de parqueo basados en el Decreto 1538 de 2005, determinando las condiciones para las zonas residenciales, comerciales e industriales, teniendo en cuenta el manual de accesibilidad al medio físico y al transporte. Es importante mencionar que este documento fue un referente nacional dado que en él se establecieron los "parámetros adecuados y definir pautas en torno a las características y condiciones necesarias para la circulación, permanencia y accesibilidad de todas las personas, y en especial a las personas con algún tipo de discapacidad, a los espacios, edificios y transporte públicos" (Presidencia de La República et. al.; 2000, p. 7). No obstante, este manual fue actualizado por la Guía de accesibilidad al espacio público y a edificaciones abiertas y de uso público (Min. de Ambiente, Vivienda y Desarrollo Territorial, 2008), en la cual se busca reducir las inconsistencias de accesibilidad.

Como se mencionó, además del transporte se aborda el espacio público en el POT, allí se contemplan disposiciones para la red vial peatonal en donde se busca la eliminación de barreras urbanísticas presentes en las condiciones superficiales de los andenes, dado que se identificó que, a las precarias dimensiones de los andenes y sus franjas de circulación y de amoblamiento se suman los problemas de irregularidad vertical y horizontal, obstáculos a nivel de franjas de circulación peatonal como gradas, muros, desniveles, postes, el cambio de texturas, la presencia de texturas lisas y la ausencia en una gran proporción de las rampas esquineras para acceso a discapacitados, entre otros (Alcaldía de Bucaramanga, 2014, p.58).

Por lo anterior, se propone como objetivos garantizar la seguridad peatonal a través de la adecuación del espacio en cuanto a diseño, pero también, se establece la necesidad de contar con señalización no solo dirigida a los automotores, sino a su vez a peatones. 1) Garantizar la seguridad del peatón con respecto al tránsito de vehículos. 2) Permitir el flujo continuo de los peatones. No se permiten obstáculos como gradas, postes, cerramientos, desniveles o cambios de texturas. 3) Garantizar las facilidades para personas de movilidad reducida $u$ otra discapacidad. 4) Contar con la señalización adecuada tanto automotores como peatones (Alcaldía de Bucaramanga, 2014, p.121).

Uno de los textos guías a nivel municipal para la adecuación del espacio ha sido el Manual de Accesibilidad al medio físico y transporte, en donde se detallan los requerimientos para lograr la accesibilidad en el espacio público, es decir, en los andenes, senderos peatonales, zonas ajardinadas, vados, escaleras, rampas y rampas escalonadas, cruces peatonales, parqueaderos, equipamiento urbano, señalización, obras en construcción; así como las de accesibilidad a edificios públicos (Presidencia de La República et al., 2000). No obstante, este referente ha generado otras barreras al homogeneizar la discapacidad.

\section{Conclusiones}

Para el caso, el modelo integrador es el más pertinente en la medida en que se centra en el análisis de los factores contextuales y de exclusión expresados en el medio físico, así como de las estrategias que desarrollan los sujetos para emanciparse, ser autónomos y avanzar hacia su entendimiento como sujetos con diversidad funcional.

El espacio público, relación social central en las ciudades, debe ser apropiado por todos los ciudadanos, incluyendo aquellos en situación de discapacidad, garantizando la accesibilidad, la movilidad y el uso equitativo del espacio como dispositivo para evitar la exclusión y la inequidad.

Apropiarse del espacio público es un derecho de los pobladores. No obstante, su uso no siempre se garantiza de igual forma especialmente cuando ciertas minorías, como las personas con discapacidad, que han sido segregadas como diferentes, tanto en el espacio como en las interacciones sociales. El ordenamiento, en un marco más reflexivo y comprensivo de esas diferencias, constituye una importante herramienta para la inclusión social entendiendo los usos, significados, infraestructura y procesos de ocupación, nuevos e históricos, del espacio público y el sentido que para las personas este tiene en sus vidas cotidianas. 
En términos generales se ha avanzado en el ámbito internacional y nacional, al menos desde el punto de vista normativo y político, en la construcción de marcos y compromisos de equidad y de derechos de las PCD. Colombia ha avanzado también en ello, aunque existe una articulación a nivel nacional en términos de accesibilidad espacial y de diseño universal, a nivel departamental y municipal no se trazaron acciones en torno a estas dimensiones en los planes de desarrollo. Sin embargo, a nivel de ordenamiento territorial el plan departamental, el metropolitano y el municipal consideran medidas orientadas a la inclusión de las personas con discapacidad.

Es importante destacar que las adecuaciones en el espacio público expresan cómo se tiende a generalizar la discapacidad y, en general, se orientan a brindar acceso a sujetos con movilidad reducida o dificultades visuales, desconociendo las singularidades propias de las personas con diversidad funcional.

\section{Referencias}

Abellán, Antonio, y Ana Olivera. (2004). Dificultades en el entorno vivido. Disponible en: http://digital.csic. es/bitstream/10261/10506/1/g-14-3-008.pdf

Alcaldía de Bucaramanga. (2016). Plan de Desarrollo 2016-2019. Gobierno de las ciudadanas y los ciudadanos. Alcaldía de Bucaramanga. 29 de Abril. Disponible en: http://www.bucaramanga. gov.co/la-ruta/download/plan de desarrollo/ Aprobacion Plan de desarrollo 2016-2019.pdf

Alcaldía de Bucaramanga. (2014). Plan de Ordenamiento Territorial de Bucaramanga. Segunda generación. 2013 -2027. Bucaramanga: Secretaría de Planeación.

Área Metropolitana de Bucaramanga. (2011). Directrices de Ordenamiento Territorial Metropolitano. Bucaramanga: Área Metropolitana de Bucaramanga.

Baquero, Jorge. (2016). Producción territorial en el espacio público. Una aproximación desde el estudio del parque de la 93, en Bogotá, en Ciudades, Estados y Política, 2016: 11-32.

Blanco, Jorge, Luciana Bosoer, y Ricardo Apaolaza. (2014). Movilidad, Apropiación y Uso del Territorio: una aproximación a partir del caso de Buenos Aires, en XIII Coloquio Internacional de Geocrítica. El control del espacio y los espacios de control. Barcelona: Universitat de Barcelona, 5-10 de Mayo.

Borja, Jordi. (2003). La ciudad conquistada. Madrid: Alianza.

Borja, Jordi, y Zaida Muxí. (2003). El espacio público, ciudad y ciudadanía. Barcelona: Electa.
Brogna, Patricia. (2006). El nuevo paradigma de la discapacidad y el rol de los profesionales de la rehabilitación. Universidad de Murcia. EL Cisne. Disponible en http://www.um.es/discatif/ PROYECTO DISCATIF/Documentos/Brogna profesionales.pdf

CEPAL. (2014). Informe regional sobre la medición de la discapacidad. Una mirada a los procedimientos de medición de la discapacidad en América Latina y el Caribe . Santiago: CEPAL.

COLDEPORTES. (2009). Biblioteca Deportiva: Guía de diseño accesible y universal.» COLDEPORTES. http://www.coldeportes.gov.co/coldeportes/ biblioteca deportiva/guia diseno accesible universal

República de Colombia. Ley 1346 de 2009 Por medio de la cual se aprueba la "Convención sobre los Derechos de las personas con Discapacidad", adoptada por la Asamblea General de la Naciones Unidas el 13 de diciembre de 2006. Bogotá, D.C.: Congreso de Colombia, 2009.

Ley 361 de 1997. Por la cual se establecen mecanismos de integración social de las personas <en situación de discapacidad $>$ y se dictan otras disposiciones. Bogotá, D.C: Congreso de Colombia, 1997.

Ley 388 de 1997. Por la cual se modifica la Ley 9a de 1989, y la Ley $3^{\text {a }}$ de 1991 y se dictan otras disposiciones. MinAmbiente. 18 de Julio de 1997. http://www.minambiente.gov.co/images/normativa/ leyes/1997/ley 0388 1997.pdf

Ley 762 de 2002. Por la cual se aprueba la "Convención Interamericana para la Eliminación de todas las Formas de Discriminación contra las Personas con Discapacidad" . Bogotá, D.C: Congreso de Colombia, 2002.

Ley Estatutaria No. 1618 de febrero de 2013 "Por medio de la cual se establecen las disposiciones para garantizar el pleno ejercicio de los derechos de las personas con discapacidad". Bogotá, D.C.: Congreso de Colombia, 2013.

Cortés, Javier, y Eva María. Sotomayor. (2016). La Exclusión Social de las personas con discapacidad en situaciones de pobreza: el caso de los campamentos saharauis de Argelia, en Index de Enfermería. Disponible en: http://scielo. isciii.es/scielo.php?script $=$ sci $\operatorname{arttext\& pid}=S 1132-$ $12962016000200012 \& \ln g=e s \& t \operatorname{lng}=e s$.

De Lorenzo, Rafael. Discapacidad, sistemas de protección y Trabajo Social. Madrid: Alianza Editorial, 2007.

Dear, Michael, Sharon Gaber, Lois Takahashi, y Robert Wilton. (1997). Seeing people differently: the sociospatial construction of disability. Environment and Planning D: Society and Space, 1997: 455-480.

Departamento Nacional de Planeación. (2015). Plan Nacional de Desarrollo 2014-2018. Todos por 
un nuevo país. DNP. Tomo 1. Disponible en: https://colaboracion.dnp.gov.co/CDT/PND/PND\%20 2014-2018\%20Tomo\%201\%20internet.pdf

Echeverría, M, y A Rincón. (2000). Ciudad de Territorialidades. Medellín: Centro de Estudios de Hábitat Popular.

Echeverría, María. (2001). Descentrar la mirada: avizorando la ciudad como territorialidad, en Espacio y territorios: razón, pasión e imaginarios, de Gustavo Montañez, Julio Carrizosa, Normando Suárez, Ovidio Delgado y Julián Lucio, 217-249. Bogotá, DC: UNIBIBLOS - UNAL.

Gobernación de Santander. (2014). Lineamientos y Directrices de Ordenamiento Territorial del departamento de Santander. Bucaramanga: Gobernación de Santander; Universidad Santo Tomás.

(2017) Plan de Desarrollo Departamental. Santander Nos Une 2016-2019. Gobernación de Santander. Disponible en: http://www.santander.gov.co/images/ cooperacion/plan de desarollo.pdf

González, Alonso. (2010). Capacidad jurídica de las personas con discapacidad. México, D. F: Comisión Nacional de Derechos Humanos.

Harvey, David. (2008). La libertad de la ciudad, en Antípoda, 15-29.

ICONTEC. (2009) NTC 4139. Accesibilidad de las personas al medio físico. Símbolo gráfico. Características generales. Bogotá, D.C.: ICONTEC.

(2013) NTC6047. Accesibilidad al medio físico. ICONTEC. Disponible en: http://enmodoin.com/wp-content/ uploads/2015/11/ntc-6047-accesibilidad-al-mediofisico-sc-admon-publica.pdf

Lefebvre, Henri. (1969). El derecho a la ciudad. Barcelona: Península.

Martínez, Ion. (2013) Prólogo: Henri Lefebvre y los espacios de lo posible, en La producción del espacio, de Henri Lefebvre, 9-30. Madrid: Capitan Swing Libros, S.L.

Mendoza, Laura. (2013) La enseñanza del espacio público y su relación al ordenamiento territorial en la ciudad. Localidad los Mártires. Trabajo de Grado para optar el Título de Licenciatura en Educación Básica con énfasis en Ciencias Sociales. Universidad Pedagógica Nacional. Facultad de Humanidades. Disponible en: http://repositorio.pedagogica.edu.co/xmlui/ bitstream/handle/123456789/403/TE-16447. pdf? sequence $=1$

Ministerio de Ambiente, Vivienda y Desarrollo Territorial. (2005). Decreto número 1538 de 2005. Por el cual reglamenta parcialmente la ley 361 de
1997. Bogotá, D.C: Presidencia de la República de Colombia.

(2008) Serie Espacio Público: Guía de accesibilidad al espacio público y a edificaciones abiertas y de uso público. Ministerio de Ambiente, Vivienda y Desarrollo Territorial. Disponible en: https:// www.slideshare.net/MarcelFernandoGutierrez/guade-accesibilidad-al-espacio-pblico

Ministerio de Salud y Protección Social. (2014) Política Pública Nacional de Discapacidad e Inclusión Social 2013-2022. MSPS. . Disponible en: https://www.minsalud.gov.co/sites/rid/Lists/ BibliotecaDigital/RIDE/DE/PS/politica-publicadiscapacidad-2013-2022.pdf

Ministerio de Transporte. (2003). Decreto 1660. Por el cual se reglamenta la accesibilidad a los modos de transporte de la población en general y en especial de las personas con discapacidad. Bogotá, D.C.: Presidencia de la República de Colombia.

Moriña, Anabel, y Noelia Melero. (2016). Redes de apoyo sociales y académicas de estudiantes con discapacidad que contribuyen a su inclusión en la enseñanza superior, en Prisma Social, 32-59.

Naciones Unidas. (2006)Convención Internacional de los Derechos de las Personas Con Discapacidad. sitio Web de División de Política Social y Desarrollo - Discapacidad. Disponible en: http://www.un.org/disabilities/documents/ convention/convoptprot-s.pdf (último acceso: 30 de Marzo de 2017)

(1994) Normas Uniformes sobre la igualdad de oportunidades para las personas con discapacidad." Naciones Unidas: Asamblea General. 4 de Marzo de 1994. Disponible en: http://www.un.org/spanish/disabilities/standardrules. pdf

(2009) Realización de los Objetivos de Desarrollo del Milenio para las personas con discapacidad mediante la aplicación del Programa de Acción Mundial para las Personas con Discapacidad y la Convención sobre los derechos de las personas con discapacidad ." Naciones Unidas. Asamblea General. Informe del Secretario General. 27 de Julio de 2009. Disponible en: http://www.un.org/ es/comun/docs/?symbol=A/64/180

Olivera, Ana. (2006). Discapacidad, accesibilidad y espacio excluyente. Una perspectiva desde la Geografía Social Urbana. Treballs de la Societat Catalana de Geografia, 2006: 326-343.

OMS. (2001) Clasificación Internacional del Funcionamiento, de la Discapacidad y de 
la Salud: CIF. Organización Mundial de la Salud. Disponible en: http://apps.who.int/iris/ bitstream/10665/43360/1/9241545445 spa.pdf

OMS. (2011). Informe mundial sobre la Discapacidad. Malta: OMS.

ONU HÁBITAT. (2017). Conferencia de las Naciones Unidas sobre la Vivienda y el Desarrollo Urbano Sostenible (Hábitat III): La Nueva Agenda Urbana. ONU HÁBITAT. http://habitat3. org/wp-content/uploads/NUA-Spanish.pdf

—. «Los Objetivos de Desarrollo Sostenible y la Iniciativa de Ciudades Prósperas.» ONU HÁBITAT. 2017. Disponible en: https://unhabitat.org/downloads/ es/mexico/cpi1603/ODS CPI.pdf

Organización de las Naciones Unidas. (s. f.). Historia de la discapacidad y las Naciones Unidas - Los primeros años: 1945 - 1955. Disponible en: http://www.un.org/spanish/disabilities/default. asp?id=572.

Presidencia de La República; Mintransporte; Mindesarrollo \& UNAL. (2000). Accesibilidad al medio físico y al transporte. Bogotá: UNAL.

Pujadas, Romà, y Jaume Font. (1998). Ordenación y planificación territorial. Madrid: Síntesis.

República de Colombia. (1991) Constitución política de Colombia. Bogotá, D.C: Corte Constitucional.

Rodríguez, Susana, y Miguel Ferreira. (2010). Desde la Dis-Capacidad hacia la diversidad funcional, en Revista Internacional de Sociología, 289309.

Taccari, Daniel. (2017). Indicadores de los ODS relacionados con la discapacidad para el seguimiento global. CEPAL. División de Estadísticas. Disponible en: https://unstats. un.org/unsd/demographic-social/meetings/2017/ chile--disability-meeting/Session\%202/ECLAC.pdf 